\title{
COMPARISON OF THE MILLER AND MACINTOSH LARYNGOSCOPES IN SIMULATED PEDIATRIC TRAUMA PATIENT: A PILOT STUDY
}

\author{
Marzena Wojewodzka-Zelezniakowicz ${ }^{1}$, Agnieszka Madziala ${ }^{2}$, Marcin Madziala ${ }^{3}$ \\ 'Department of Emergency Medicine and Disaster, Medical University of Białystok, Poland \\ ${ }^{2}$ Polish Society of Disaster Medicine, Warsaw, Poland \\ ${ }^{3}$ Department of Emergency Medicine, Medical University of Warsaw, Poland
}

\begin{abstract}
INTRODUCTION: Airway management in pediatric trauma patients is challenging. Direct laryngoscopy is the gold standard for endotracheal intubation in emergency and trauma patients. The aim of the present study was to evaluate the performance of Miller (MIL) and Macintosh (MAC) laryngoscopes when employed in emergency pediatric intubation scenarios.
\end{abstract}

METHODS: This was a prospective, randomized, crossover, single-center study on novice physicians recruited on a voluntary basis. Each participant performer endotracheal intubation using Miller or Macintosh laryngoscopes during two airway scenarios: Scenario A - normal airway; Scenario B - difficult airway, defined as scenario in which the patient was placed on backboard with neck immobilization performer using rigid cervical collar. The order of use of one or other of the devices was randomized with a ratio of 1:1. The primary endpoint was the first attempt success rate.

RESULTS: The effectiveness of the first intubation attempt in Scenario B for MAC was $36.8 \%$, for MIL - 44.7\%; while in Scenario A for MAC this was 36.8\%, for MIL - 44.7\%. During Scenario A, the median duration time of intubation using MIL was $24.5 \mathrm{~s}$ [IQR; 21-32.5] and $23 \mathrm{~s}$ [IQR; 20.5-31] for MAC. During Scenario B, median intubation time with the MAC laryngoscope was 40.5 [IQR; 36.5-47] s, and with MIL 37.5 [IQR; 33-44.5] seconds.

CONCLUSIONS: We concluded that in trauma pediatric patients the Miller laryngoscope is associated with higher first attempt success rates than the Macintosh laryngoscope. These data suggest that for patients with cervical spine immobilization, the Miller laryngoscope should be the preferred method of intubation in emergency medicine conditions.

KEY WORDS: endotracheal intubation, simulation, pediatric, trauma, efficacy

Disaster Emerg Med J 2017; (2)1: 1-6

\section{INTRODUCTION}

Securing a clear airway in children compared with adults can be a challenge for medical staff due to the anatomical differences present, including a shorter neck, a relatively larger head or larger and more cranially located epiglottis [1, 2]. During a laryngoscopy, the larynx, both in young children and infants, often seems to be located more anteriorly [3]. In this situation it may be helpful to keep the head tilted and to shift the tongue to the left out of the line of sight. However, such maneuvers within the cervical spine are accepted only in non-traumatic patients. In the case of trauma patients (both adults and children), the immobilization of the cervical spine in order to reduce the possibility of injury is essential [4-6]. This limitation could result in the worsening of glottis visibility and less effective endotracheal intubation $[5,6]$. During endotracheal 
intubation in children, most health professionals commencing endotracheal intubation in a direct laryngoscopy use a straight Miller laryngoscope blade. However, as indicated in the scientific literature, in children above 2 years of age the curved Macintosh blade may also be used [7-9]. However, regardless to the chosen method of the endotracheal intubation in pediatric patients, it should be noted that intubation should be done by the most experienced team member as more than three or four unsuccessful intubation attempts may result in bleeding and edema and eventually lead to the phenomenon in which each subsequent intubation attempt increases the bleeding and swelling, leading to a situation defined by the Difficult Airway Society as "cannot intubate, cannot ventilate".

The aim of this study was to compare the efficacy of endotracheal intubation using Macintosh and Miller laryngoscopes in simulated cervical trauma pediatric patient conditions.

\section{MATERIAL AND METHODS}

\section{Study design}

Approval from the Institutional Review Board of the Polish Society for Disaster Medicine (Approval no. 21/04/2016.IRB) and verbal informed consent from each participant were obtained before the study. Physicians included in the study were recruited on a voluntary basis. Inclusion criteria were that they had to be graduate physicians completing a postgraduate internship course in Emergency Medicine. This prospective, randomized, cross-over manikin study was performed in July 2016. Brief standardized prior training was given to each physician. The study devices consisted of the Miller laryngoscope (MIL) with a size 2 blade and the Macintosh laryngoscope (MAC) also with a size 2 blade (Fig. 1). We used a 5.0 cuffed endotracheal tube (ETT) for each intubation attempt. We also use a 6 French outer diameter malleable Slick stylet (Teleflex Medical, Research Triangle Park, NC, USA).

The participants performed endotracheal intubations with each device in two scenarios:

- Scenario A: normal airway scenario;

- Scenario B: difficult airway scenario. To simulate a trauma patient, manikin was placed on the board, and a hard cervical collar was applied to the manikin with its head fixed in a neutral position (Fig. 2).
(A)

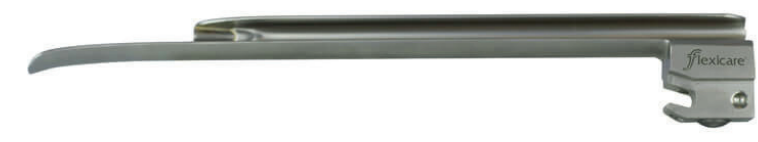

(B)

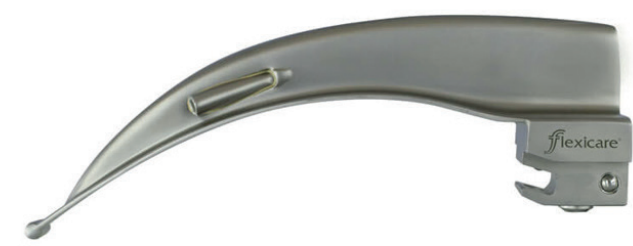

FIGURE 1. Laryngoscope blades used in the study: (A) Miller blade; (B) Macintosh blade

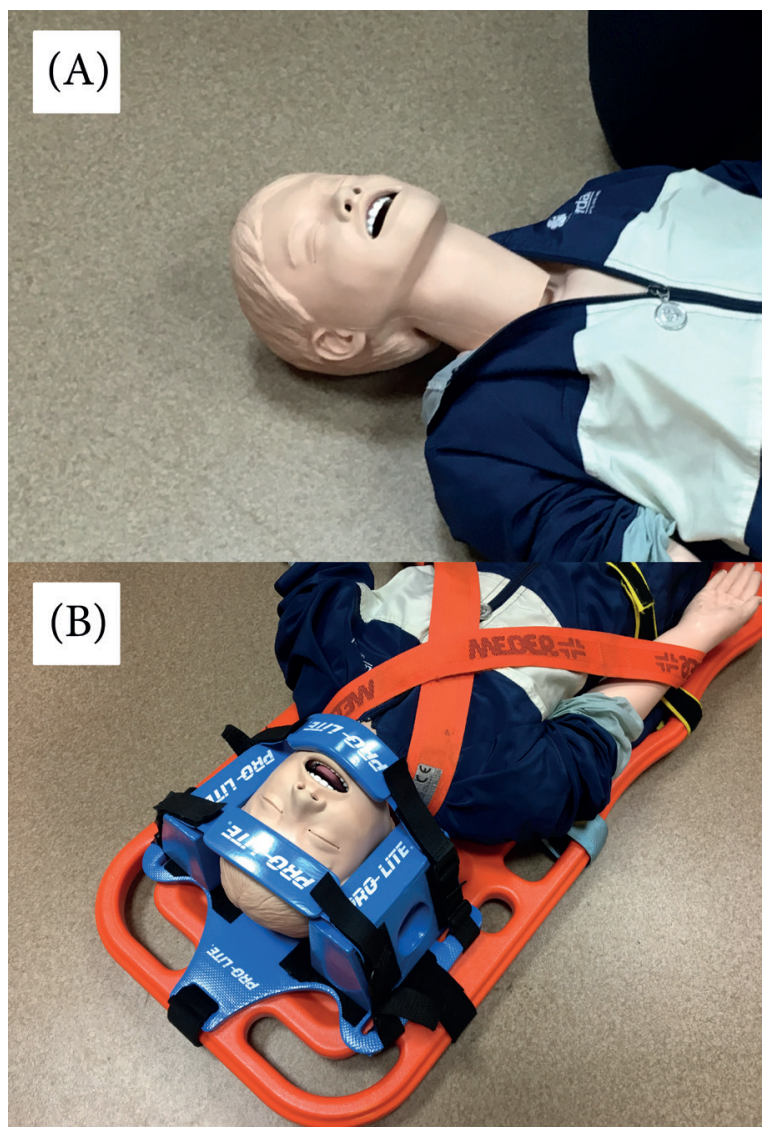

FIGURE 2. Scenarios used in the study: (A) normal airway scenario; (B) difficult airway scenario

To simulate the scenario of a multi-trauma patient, a standardized airway manikin with a regular airway was used (MegaCode Kelly ${ }^{\mathrm{Tw}}$; Laerdal, Stavanger, Norway).

Each participant performed a total of 10 intubation attempts with the two intubation devices in a normal airway scenario. The order of partici- 
pants and intubation devices were random. For this purpose, we use Research Randomizer software to divide participants into four groups. The first group started with MIL in Scenario A, with the second using MIL in Scenario $B$, the third using MAC in Scenario $A$, and the fourth using MAC in Scenario $B$ (Fig. 3). Participants were offered the maximum one intubation attempt with each device. After performing the procedure, participants had 10 minutes break for a rest, and then performed an intubation using another technique.

\section{Outcomes}

The primary endpoint was time to intubation (TTI), defined as the time in seconds from insertion of the device between the teeth to first ventilation. Successful intubation was verified when the lungs were inflatable by an Ambu bag. Failed intubation was defined as when the trachea was not intubated in the first attempt or the intubation attempt required more than $60 \mathrm{~s}[10,11]$. After each scenario, the participants were asked to grade glottis visualization using the Cormack-Lehane laryngoscopic grading scale (Grade 1 to Grade 4) [12], and to mark the ease of intubation on a 10-point visual analog scale (VAS; 1 - very difficult; 10 — very easy).

\section{Statistical analysis}

All procedures were recorded using a GoPro HERO 5 camera (GoPro GmbH, Munich, Germany), and all of the time variables were precisely analyzed by reviewing the recorded data. Statistical calculations were performer using the Statistica 12.5EN statistical software (StatSoft, Tulsa, OK, USA). Values of $p<0.05$ were considered significant. Categorical variables were presented as frequencies,

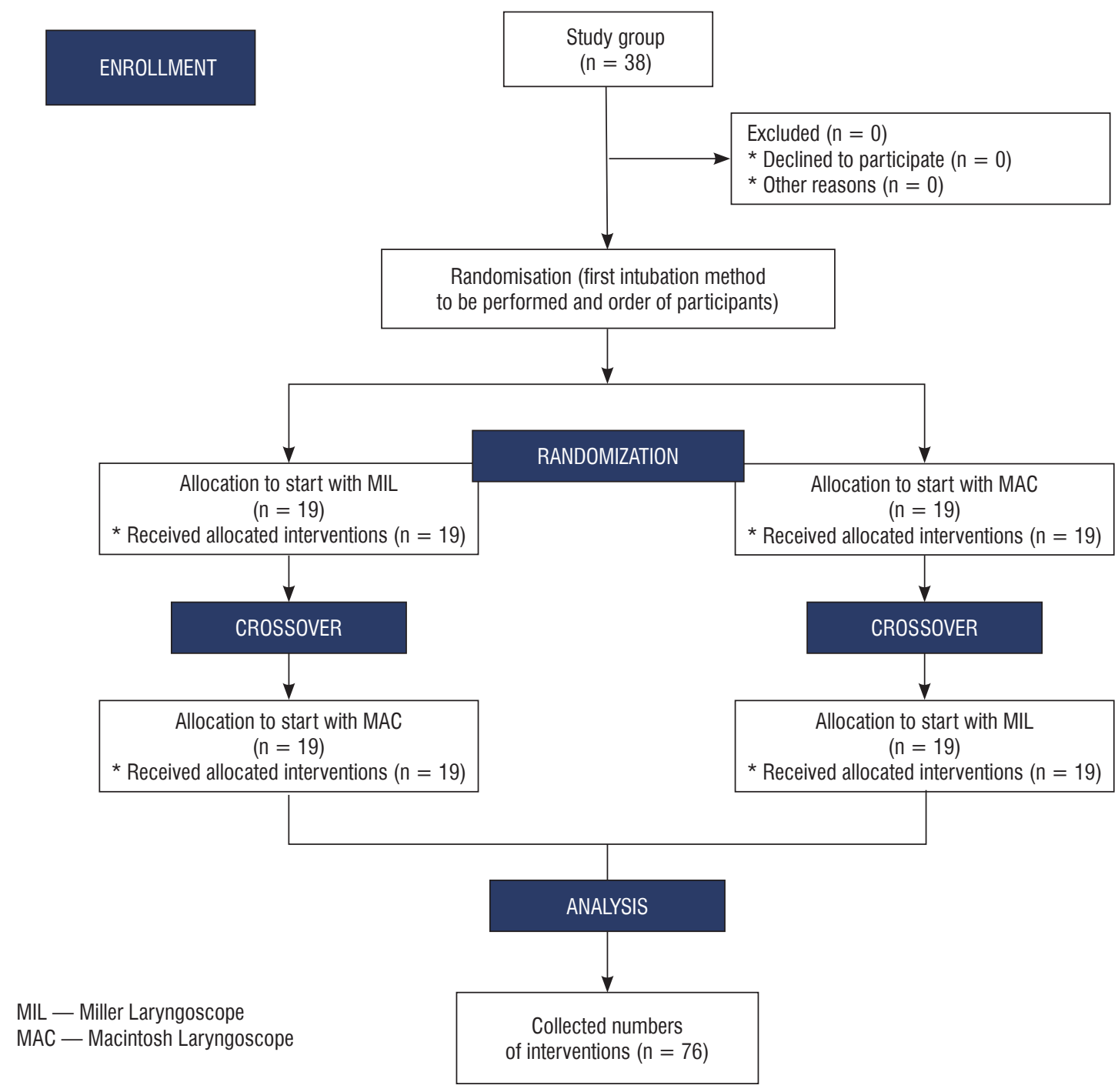

FIGURE 3. CONSORT (Consolidated Standards of Reporting Trials) flow diagram 
and continuous and ordinal variables as medians with an interquartile range (IQR). We compared qualitative variables by using Fisher's exact test and the Kruskal-Willis test, and the quantitative variables by using Student's test. All statistical tests were two-sided.

\section{RESULTS}

The study involved 38 novice physicians participating in a compulsory course in emergency medicine. All participants declared their ability to perform endotracheal intubation using direct laryngoscopy for both adults and pediatric patients. The median age of participants was 25 [IQR; 24.5-26] years.

\section{Normal airway scenario}

During the endotracheal intubation in normal airways (Scenario A), the effectiveness of the first endotracheal intubation attempt using MIL and MAC was varied and amounted to $76.3 \%$ vs. $78.9 \%$, respectively (Tab. 1). The median duration time of intubation using MIL was $24.5 \mathrm{~s}$ [IQR; 21-32.5] and was slightly shorter compared to the MAC 23 s [IQR; 20.5-31] (Fig. 4). Visualization of the glottis, as well as the ease of intubation were comparable between these two devices.

\section{Difficult airway scenario}

In difficult airways scenario where the manikin was placed on the spinal board and a rigid cervical collar used, the effectiveness of the first intubation attempt for MAC was 36.8\%, and for MIL - 44.7\%. The difference in the effectiveness of the first attempted intubation was statistically significant $(p=0.023)$. The median duration of intubation during the Scenario B with the MAC laryngoscope was $40.5 \mathrm{~s}$ [IQR; 36.5-47], and with MIL $37.5 \mathrm{~s}$ [IQR; 33-44.5] ( $p=0.011)$. The endotracheal intubation using MIL was associated with better visualizing of the glottis compared to the Macintosh laryngoscope



FIGURE 4. Median intubation time

(Tab. 2). A similar relationship was observed when the scale of ease of the implementation of the procedure was analyzed (VAS).

\section{DISCUSSION}

The study demonstrated that cervical spine immobilization during simulated endotracheal intubation using a laryngoscope with the Miller blade was associated with higher efficiency than intubation with the Macintosh laryngoscope.

Landy and Nossaman indicated that laryngoscopy with the Miller blade is highly effective and safe in patients undergoing general anesthesia for elective surgery with a low incidence of difficult laryngoscopy and difficult orotracheal intubation [13]. Varghese and Kundu, evaluating intubation among twenty children aged 1-24 months, indicated that the Miller and the Macintosh blades provide similar laryngoscopic views and intubating conditions. Verghese and Kundu's study showed that

\begin{tabular}{|c|c|c|c|}
\hline Variable & Miller laryngoscope & Macintosh laryngoscope & P-value \\
\hline First attempt success rate & $76.3 \%$ & $78.9 \%$ & $p=0.775$ \\
\hline Time to intubation (s) & 24.5 [IQR; 21-32.5] & 23 [IQR; 20.5-31] & $p=0.658$ \\
\hline $\begin{array}{l}\text { Cormack \& Lehane grade } \\
1 \\
2 \\
3 \\
4\end{array}$ & $\begin{array}{c}30(78.9 \%) \\
8(21.1 \%) \\
- \\
-\end{array}$ & $\begin{array}{c}28 \\
10(26.4 \%) \\
- \\
-\end{array}$ & $p=0.855$ \\
\hline VAS score & $6[\mathrm{IQR} ; 5.5-8]$ & $5.5[\mathrm{IQR} ; 5-7.5]$ & $p=0.44$ \\
\hline
\end{tabular}




\begin{tabular}{|l|c|c|c|}
\hline \multicolumn{1}{|c|}{ Table 2. Intubation during scenario A } \\
\hline \multicolumn{1}{|c|}{ Variable } & Miller laryngoscope & Macintosh laryngoscope & P-value \\
\hline First attempt success rate & $44.7 \%$ & $36.8 \%$ & $\mathrm{p}=0.045$ \\
\hline Time to intubation (s) & $37.5[\mathrm{IQR} ; 33-44.5]$ & $40.5[\mathrm{IQR} ; 36.5-47]$ & $\mathrm{p}=0.011$ \\
\hline Cormack \& Lehane grade & & & $\mathrm{p}=0.023$ \\
1 & $4(10.5 \%)$ & $21(58.3 \%)$ & \\
2 & $23(60.5 \%)$ & $16(39.1 \%)$ & \\
3 & $11(29 \%)$ & $1(2.6 \%)$ & \\
4 & - & $3.5[\mathrm{IQR} ; 3-5]$ & $\mathrm{p}=0.013$ \\
\hline VAS score & $5[\mathrm{IQR} ; 3.5-6]$ & & \\
\hline
\end{tabular}

when a limited view is obtained, a change of blade provided a better glottis view. Placing the tip of the Miller blade in the vallecula provides satisfactory intubating conditions in this age group [7]. The advantage of the Macintosh compared to Miller blade in children under 2 years of age has also been indicated by other authors $[9,14]$. In our study we analyzed endotracheal intubation with the use of a manikin presenting a 6 -year-old child. In their study, Inal et al. [15] compared the efficacy of endotracheal intubation using the Miller laryngoscope with the TruView EVO2 optical laryngoscope. The study was conducted on 50 2-8-year-old pediatric patients presenting for surgery requiring endotracheal intubation. Their results suggest that when compared to the Miller laryngoscope, the TruView EVO2 laryngoscope appears to improve the view of the glottis although it requires a longer time for tracheal intubation to be performed. However, their Intubation Difficulty Scores (IDS) were similar [15].

Many studies have shown the prevalence of videolaryngoscopy compared with direct laryngoscopy in different clinical situations [16-19]. In studies published by Szarpak et al., the use of videolaryngoscopy compared with direct laryngoscopy during endotracheal intubation in children under simulated CPR settings was associated with a higher efficiency of the first intubation attempt and a shorter procedure time $[20,21]$. In turn, Eisenberg et al., in a retrospective cohort study on endotracheal intubations in children aged 0-18 years in a pediatric ED between 2004 and 2014, examined 452 endotracheal intubations and which showed no difference between direct laryngoscopy and videolaryngoscopy with regard to first-pass intubation success rate, complication rate, and successful intubation rate by Emergency Department health care professionals in children undergoing endotracheal intubation in a pediatric Emergency Department
[22]. In turn, Hippard et al. showed higher success rates and shorter intubation times with the Miller blade compared to either videolaryngoscope which may reflect one's greater experience in direct laryngoscopy [23]. However, due to the availability of the laryngoscopes with Miller or Macintosh blades, these devices are most often used in Poland, both in emergency departments and by Emergency Medical Service teams [4].

Our study has several limitations. First we used a standard manikin with a standard anatomical design, which might not adequately simulate emergency clinic conditions. However, only the use of manikin allows one to perform randomized crossover trials on the participants with limited experience in the field of clinical endotracheal intubation. Simulated conditions allow one to perform a study without prejudice to the health of potential patients. Secondly, the participants knew the scenario before evaluation. Thirdly, the severity of dental trauma was not considered.

The main strengths of our study are the number of novice physicians included in this trial and the randomized, crossover design of this study.

\section{CONCLUSIONS}

We conclude that endotracheal intubation with the use of the Miller laryngoscope in trauma pediatric patients is associated with higher first attempt success rates than the Macintosh laryngoscope. Our results suggest that for patients with cervical spine immobilization, the Miller laryngoscope should be the preferred method of intubation in emergency medicine conditions.

Acknowledgement: We are grateful to all study participants.

Conflict of interest: None declared. 


\section{REFERENCES}

1. Andersen LW, Raymond TT, Berg RA, et al. American Heart Association's Get With The Guidelines-Resuscitation Investigators. Association Between Tracheal Intubation During Pediatric In-Hospital Cardiac Arrest and Survival. JAMA. 2016; 316(17): 1786-1797, doi: 10.1001/ jama.2016.14486, indexed in Pubmed: 27701623.

2. Hansen M, Meckler G, 0 Brien K, et al. Pediatric Airway Management and Prehospital Patient Safety: Results of a National Delphi Survey by the Children's Safety Initiative-Emergency Medical Services for Children. Pediatr Emerg Care. 2016; 32(9): 603-607, doi: 10.1097/ PEC.0000000000000742, indexed in Pubmed: 27253653.

3. Szarpak L, Karczewska K, Evrin T, et al. Comparison of intubation through the McGrath MAC, GlideScope, AirTraq, and Miller Laryngoscope by paramedics during child CPR: a randomized crossover manikin trial. Am J Emerg Med. 2015; 33(7): 946-950, doi: 10.1016/j. ajem.2015.04.017, indexed in Pubmed: 25937380.

4. Gawlowski P, Smereka J, Madziala M, et al. Comparison of the Macintosh laryngoscope and blind intubation via the iGEL for intubation with cervical spine immobilization: A randomized, crossover, manikin trial. Am J Emerg Med. 2016 [Epub ahead of print], doi: 10.1016/j. ajem.2016.11.064, indexed in Pubmed: 28041757.

5. Truszewski Z, Szarpak L, Czyzewski L, et al. A comparison of the ETView VivaSight SL against a fiberoptic bronchoscope for nasotracheal intubation of multitrauma patients during resuscitation. A randomized, crossover, manikin study. Am J Emerg Med. 2015; 33(8): 1097-1099, doi: 10.1016/j.ajem.2015.04.078, indexed in Pubmed: 25983119.

6. Bogdański Ł, Truszewski Z, Kurowski A, et al. Simulated endotracheal intubation of a patient with cervical spine immobilization during resuscitation: a randomized comparison of the Pentax AWS, the Airtraq, and the McCoy Laryngoscopes. Am J Emerg Med. 2015; 33(12): 1814-1817, doi: 10.1016/j.ajem.2015.09.005, indexed in Pubmed: 26494629.

7. Varghese E, Kundu R. Does the Miller blade truly provide a better laryngoscopic view and intubating conditions than the Macintosh blade in small children? Paediatr Anaesth. 2014; 24(8): 825-829, doi: 10.1111/pan.12394, indexed in Pubmed: 24690084.

8. Sims C. The Miller and Macintosh blades in young children. Paediatr Anaesth. 2014; 24(11): 1196, doi: 10.1111/pan.12540, indexed in Pubmed: 25279681.

9. Kulkarni AP, Tirmanwar AS. Comparison of glottic visualisation and ease of intubation with different laryngoscope blades. Indian J Anaesth. 2013; 57(2): 170-174, doi: 10.4103/0019-5049.111846, indexed in Pubmed: 23825817.

10. Truszewski Z, Krajewski P, Fudalej M, et al. A comparison of a traditional endotracheal tube versus ETView SL in endotracheal intubation during different emergency conditions: A randomized, crossover cadaver trial. Medicine (Baltimore). 2016; 95(44): 1-6, doi: 10.1097/ MD.0000000000005170, indexed in Pubmed: 27858851.

11. Truszewski Z, Czyzewski L, Smereka J, et al. Ability of paramedics to perform endotracheal intubation during continuous chest compressions: a randomized cadaver study comparing Pentax AWS and
Macintosh laryngoscopes. Am J Emerg Med. 2016; 34(9): 1835-1839, doi: 10.1016/j.ajem.2016.06.054, indexed in Pubmed: 27369468.

12. Cormack RS, Lehane J. Difficult tracheal intubation in obstetrics. Anaesthesia. 1984; 39(11): 1105-1111, indexed in Pubmed: 6507827.

13. Landry WB, Nossaman BD. Airway risk factors for the Miller laryngoscope blade. J Clin Anesth. 2016; 33: 62-67, doi: 10.1016/j. jclinane.2016.01.032, indexed in Pubmed: 27555135.

14. Passi Y, Sathyamoorthy M, Lerman J, et al. Comparison of the laryngoscopy views with the size 1 Miller and Macintosh laryngoscope blades lifting the epiglottis or the base of the tongue in infants and children $<2$ yr of age. Br J Anaesth. 2014; 113(5): 869-874, doi: 10.1093/ bja/aeu228, indexed in Pubmed: 25062740.

15. Inal MT, Memis D, Kargi M, et al. Comparison of TruView EVO2 with Miller laryngoscope in paediatric patients. Eur J Anaesthesiol. 2010; 27(11): 950-954, doi: 10.1097/EJA.0b013e32833f539f, indexed in Pubmed: 20829701.

16. Singh $R$, Singh $P$, Vajifdar H. A comparison of Truview infant EVO2 laryngoscope with the Miller blade in neonates and infants. Paediatr Anaesth. 2009; 19(4): 338-342, doi: 10.1111/j.14609592.2009.02929.x, indexed in Pubmed: 19335346.

17. Goksu E, Kilic T, Yildiz G, et al. Comparison of the C-MAC video laryngoscope to the Macintosh laryngoscope for intubation of blunt trauma patients in the ED. Turk J Emerg Med. 2016; 16(2): 53-56, doi: 10.1016/j.tjem.2016.02.001, indexed in Pubmed: 27896321.

18. Yumul R, Elvir-Lazo OL, White PF, et al. Comparison of three video laryngoscopy devices to direct laryngoscopy for intubating obese patients: a randomized controlled trial. J Clin Anesth. 2016; 31: 71-77, doi: 10.1016/j.jclinane.2015.12.042, indexed in Pubmed: 27185681.

19. Lewis $S R$, Butler $A R$, Parker J, et al. Videolaryngoscopy versus direct laryngoscopy for adult patients requiring tracheal intubation. Cochrane Database Syst Rev. 2016; 11: CD011136, doi: 10.1002/14651858. CD011136.pub2, indexed in Pubmed: 27844477.

20. Szarpak L, Truszewski Z, Czyzewski L, et al. A comparison of the McGrath-MAC and Macintosh laryngoscopes for child tracheal intubation during resuscitation by paramedics. A randomized, crossover, manikin study. Am J Emerg Med. 2016; 34(8): 1338-1341, doi: 10.1016/j. ajem.2015.11.060, indexed in Pubmed: 26712571.

21. Szarpak Ł, Czyżewski Ł, Kurowski A, et al. Comparison of the TruView $P C D$ video laryngoscope and macintosh laryngoscope for pediatric tracheal intubation by novice paramedics: a randomized crossover simulation trial. Eur J Pediatr. 2015; 174(10): 1325-1332, doi: 10.1007/ s00431-015-2538-0, indexed in Pubmed: 25894914.

22. Eisenberg MA, Green-Hopkins I, Werner H, et al. Comparison Between Direct and Video-assisted Laryngoscopy for Intubations in a Pediatric Emergency Department. Acad Emerg Med. 2016; 23(8): 870-877, doi: 10.1111/acem.13015, indexed in Pubmed: 27208690.

23. Hippard HK, Kalyani G, Olutoye $O A$, et al. A comparison of the Truview PCD and the GlideScope Cobalt AVL video-laryngoscopes to the Miller blade for successfully intubating manikins simulating normal and difficult pediatric airways. Paediatr Anaesth. 2016; 26(6): 613-620, doi: 10.1111/pan.12906, indexed in Pubmed: 27083381. 